Images du travail, travail des images

$8 \mid 2020$

Filmer, travailler, chercher

\title{
Filmer, enquêter, montrer : allers et retours
}

To film, to show, to do fieldwork : round trips

\section{Annalisa Lendaro}

\section{OpenEdition}

\section{Journals}

Édition électronique

URL : http://journals.openedition.org/itti/369

DOI : 10.4000/itti.369

\section{Éditeur}

Université de Poitiers

\section{Référence électronique}

Annalisa Lendaro, "Filmer, enquêter, montrer : allers et retours », Images du travail, travail des images

[En ligne], 8| 2020, mis en ligne le 01 février 2020, consulté le 14 avril 2021. URL : http://

journals.openedition.org/itti/369; DOI : https://doi.org/10.4000/itti.369

Ce document a été généré automatiquement le 14 avril 2021.

Images du travail, travail des images 


\section{Filmer, enquêter, montrer : allers et retours}

To film, to show, to do fieldwork : round trips

Annalisa Lendaro

\section{Introduction}

1 Benvenuti $(53$ ', 2017) est un film documentaire issu d'une recherche qualitative conduite entre 2013 et 2016 par Laura Auriole, documentariste et anthropologue de formation, et moi-même, sociologue. Le protagoniste du film est Lampedusa, une petite île italienne de seulement $20 \mathrm{~km} 2$, connue pour être la destination de milliers de migrants qui, chaque année, risquent leur vie en traversant la Méditerranée pour rejoindre l'Europe. Elle est frontière pour les uns et paradis balnéaire pour d'autres, car ses eaux turquoises, ses paysages paradisiaques, la nonchalance apparente de ses habitants en font aussi une destination touristique prisée : les contrastes entre ces mondes qui ne font que s'effleurer sont au centre de la focale, à la fois de la recherche et du film.

2 Dans cette contribution, je m'interrogerai sur ce que le fait de filmer a produit sur les différentes étapes de cette recherche. Ce texte sera ainsi l'occasion de revenir de manière réflexive non seulement sur les effets de cette aventure à deux sur ma propre pratique de recherche en sociologie, mais aussi sur les conséquences engendrées par les tensions et les nécessaires compromis, tant au niveau des façons de faire de la recherche que de son produit, en l'occurrence, le film.

Dans la première partie de l'article, je reconstituerai de manière chronologique les étapes qui ont mené à la réalisation du film : d'abord, il sera question des motivations qui ont poussé une chercheuse profane à s'aventurer sur un terrain nouveau, celui du cinéma documentaire ; ensuite, j'expliciterai quels ont été les enjeux liés au travail en binôme, constamment ponctué de choix impactant la manière de faire de la recherche pour l'une, et de faire du cinéma pour l'autre ; enfin, je reviendrai sur la phase de post- 
production, en mettant l'accent sur les négociations parfois difficiles avec la production autour de ce qui « fait science » et de ce qui est considéré incompatible avec elle.

Dans la deuxième partie de la contribution, j'approfondirai le thème du film comme outil pour la recherche "en train de se faire" (et non pas comme produit d'une recherche achevée). Plus spécifiquement, il sera question de mettre en lumière les potentialités du film dans le cadre du prolongement du terrain d'enquête. Je discuterai l'intérêt méthodologique de la projection du film dans le cadre d'entretiens collectifs avec les habitants de l'île de Lampedusa, réalisés entre avril et juin 2018 lors d'un retour sur ce terrain quatre ans après le dernier tournage. J'expliciterai de quelle manière cette pratique peut utilement se combiner avec les "piliers méthodologiques " de l'enquête qualitative que sont l'entretien compréhensif et l'observation.

Pour conclure, j'insisterai plus particulièrement sur l'opportunité pour le chercheur de proposer un droit de regard aux personnes concernées par le contenu du film. L'intérêt de la démarche est à mon sens double : d'une part, cela revient à prendre au sérieux le devoir de restitution des résultats de l'enquête et à traduire en actes une certaine éthique du travail de chercheur ; d'autre part, cet usage du film contribue à déclencher une discussion collective sur les choix des réalisatrices (d'interprétation d'énoncés d'autrui, de restitution à l'image de situations, etc.) qui peut permettre non seulement d'affiner la compréhension des phénomènes déjà étudiés précédemment, mais aussi de favoriser l'émergence de nouvelles pistes de recherche.

\section{Les origines : l'exigence de " montrer", et ses liens avec l'engagement dans la recherche}

6 Au printemps 2013, cela faisait déjà quelques années que mon intérêt s'était porté sur les migrations contemporaines, les inégalités en matière de droit à la mobilité internationale, les outils de la gestion morale et matérielle de la frontière et leurs conséquences socio-politiques tout autant sur les personnes tentant de la traverser que sur les sociétés dites d'accueil.

7 À ce moment de ma carrière de jeune chercheuse, le fruit de mes enquêtes et réflexions prenait principalement la forme d'articles dans des revues académiques et de communications dans des colloques. Néanmoins, j'avais conscience que ma recherche touchait un public restreint, celui des collègues, souvent au fait des enjeux contemporains au cœur de mes enquêtes. Je ne saurais dire grâce à qui ou à quoi, mais cette prise de conscience a été à l'origine d'une insatisfaction qui a été à son tour le moteur de ce projet de film documentaire. Cette insatisfaction tenait à l'incapacité de pouvoir communiquer (et discuter) des résultats de la recherche en sciences sociales à un public élargi, et ce d'autant plus que l'information médiatique concernant les problématiques migratoires est généralement lacunaire et sensationnaliste, parfois clairement erronée et orientée.

8 Minuscule île italienne située à seulement 130 kilomètres des côtes tunisiennes, Lampedusa était périodiquement sous les feux des médias depuis au moins une dizaine d'années. Sa notoriété internationale s'est construite progressivement suivant un processus de «spectacularisation » de la frontière (Cuttitta 2012, 2016) construit par une couverture médiatique intensive et largement instrumentalisée d'événements 
dramatiques, dont les naufrages en Méditerranée et les révoltes qui ont eu lieu au sein du centre de rétention pour migrants de l'île.

Parallèlement, les mêmes médias mettaient parfois en exergue le caractère remarquablement accueillant et bienveillant de la population de Lampedusa vis-à-vis de migrants appelés tout autant "clandestins", "extracommunautaires", ou « irréguliers »: forces de l'ordre et habitants pouvaient alors être présentés dans la presse ou dans certaines productions cinématographiques comme un binôme efficace et complémentaire dans la gestion d'une «urgence migratoire» naturalisée et décontextualisée. Ces généralisations et allant de soi constituent évidemment autant de simplifications.

10 J'ai souhaité me rendre sur place afin de donner non seulement de la profondeur scientifique à ce tableau mais aussi de donner à comprendre la complexité de la situation au grand public qui n'a généralement accès qu'aux informations transmises par les médias. Aussi, afin de rendre compte des effets de la gestion sécuritaire de la frontière contemporaine, à la fois sur les personnes en migration et sur les résidents de cette île, il m'a paru nécessaire de ne pas miser uniquement sur l'écrit et j'ai proposé à une amie documentariste de m'accompagner dans cette découverte. En acceptant ma proposition, elle me permettait d'envisager de satisfaire mon désir d'assumer une forme d'engagement politique à mes yeux compatible avec la démarche sociologique ${ }^{1}$. Car, dès cette époque, il me semblait qu'un film de recherche, soucieux de scientificité tout en restant accessible au grand public, pourrait contrecarrer certains discours politiquement dangereux ${ }^{2}$. J'avais également le sentiment que le film pouvait permettre d'exprimer certaines idées de manière plus explicite que l'écrit scientifique, notamment grâce aux récits des personnes filmées.

11 La suite de l'expérience m'a montré que les choses étaient toutes bien plus compliquées que ce que j'avais pu imaginer. Cependant la question du public touché par un film de recherche reste centrale quant aux finalités de la démarche : réussir à dialoguer avec des non spécialistes autour des droits et des libertés fondamentales ainsi que de leur non-respect dans les territoires frontaliers a été certainement possible grâce au support filmique.

\section{Les tournages : enjeux et difficultés d'un travail à deux}

12 À partir du début des années 2000, Lampedusa est progressivement devenue un symbole de la frontière européenne (Cuttitta 2015). Ainsi, cette petite île à l'extrême sud de l'Italie a été largement investie par les chercheurs en sciences sociales (Ritaine 2015, Melot 2009, Rodier 2014, Cuttitta 2012, pour ne citer qu'une petite partie des études publiées en français), qui en ont fait un terrain exemplaire pour étudier l'enfermement administratif des étrangers et souvent pour dénoncer les conséquences de la gestion de l'immigration dite irrégulière. Au départ, la recherche initiée en été 2013 avec Laura Auriole à la caméra s'inscrivait dans cette perspective. Il était notamment question d'étudier et de filmer les formes que prend la rétention administrative des étrangers sur une île frontière (les pratiques de fichage, l'aménagement de l'espace de confinement, les bricolages et les exceptions à la règle dans la mise en œuvre des procédures d'accueil, la militarisation du territoire, etc.). 
Image 1 : migrants enfermés dans le CSPA de Lampedusa

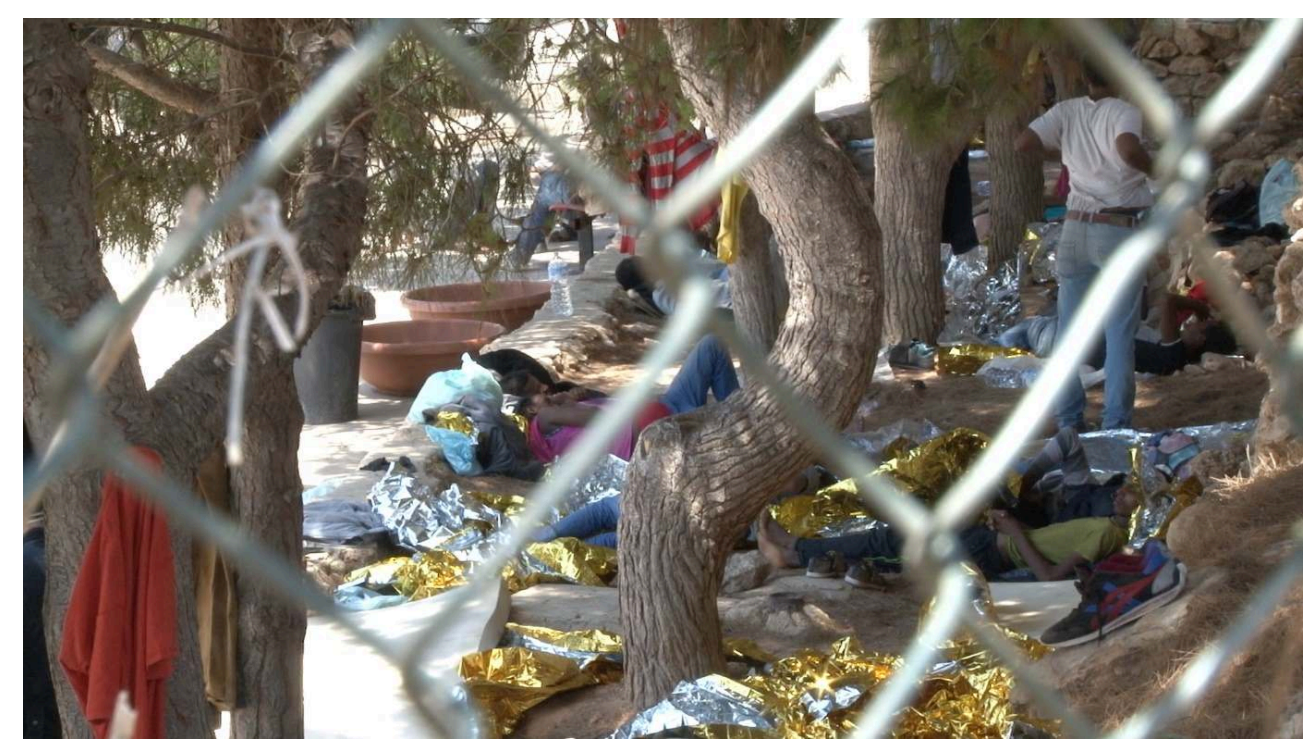

Source : Laura Auriole, juillet 2014

13 La première période de tournage, en juillet de cette même année, a été l'occasion de découvrir les lieux et les modalités de la gestion de l'immigration et du premier accueil des migrants ayant survécu à la traversée de la Méditerranée. Cependant, ce premier séjour a également été le moment d'une découverte inattendue: nous avons été frappées par la coexistence paradoxale et criante entre un système policier et militaire imposant, visible et banalisé dans l'espace public, et un tourisme estival de masse, les deux fonctionnant en parallèle de la vie ordinaire des résidents de l'île. Le contraste entre ces mondes et les frontières invisibles qui les séparent nous ont déroutées. Nous avons alors toutes deux ressenti le besoin de recueillir les témoignages de plusieurs groupes de personnes : migrants, garde-côtes, résidents et touristes.

L'idée initiale d'accorder une place particulière, dans la recherche et dans le film, à l'expérience des migrants a été assez vite reconsidérée. Lors du premier tournage, nous avons toutefois eu l'occasion de rencontrer et de discuter à plusieurs reprises avec des jeunes migrants qui pouvaient entrer et sortir aisément du Centre de Premier Secours et d'Accueil (CPSA) ${ }^{3}$. Nous avions ainsi imaginé, pour le deuxième tournage prévu au printemps 2014, de mettre en place un dispositif de suivi quotidien de quelques personnes migrantes rencontrées en dehors des murs du centre de rétention, mais le naufrage du 3 octobre 2013 (Nicolosi et Panebianco, 2015) suivi de la mise en place de l'opération Mare Nostrum ${ }^{4}$ puis, quelques semaines plus tard, de la polémique sur les conditions d'accueil dans le Centre de Premier Secours et d'Accueil (CPSA) ${ }^{5}$ ont changé radicalement le contexte du tournage. En effet, l'ensemble de ces éléments a conduit la Préfecture d'Agrigento, responsable de la gestion du CPSA, à refuser de nous donner accès à la structure - laquelle fut d'ailleurs fermée temporairement pour rénovation à partir de mars 2014 -, tandis que Lampedusa n'a plus accueilli de survivants des traversées, lesquels étant directement emmenés en Sicile jusqu'en octobre 2014 (date de fin de l'opération Mare Nostrum). De fait, lors de notre deuxième tournage en mai 2014, puis pendant le troisième et dernier qui se déroula en juillet et août de la même année, nous n'avons pu rencontrer de migrants sur l'île. 

été emprunt de doutes et de réflexions, accompagnés de nouvelles envies et de la nécessité de faire des choix. D'une part, s'est posée la question des moyens de faire financer notre projet de film : ce dernier nous paraissait de plus en plus nécessaire au vu de l'engouement médiatique autour des questions brûlantes concernant les morts en Méditerranée, l'accueil sur terre des survivants, l'indignation (sans conséquences tangibles) des personnalités politiques ou encore les diverses réactions des habitants de l'île de Lampedusa. Le besoin d'organiser un tournage hors saison touristique, en mai 2014, a révélé le décalage de statuts au sein de l'équipe: moi, chercheuse en poste, Laura, professionnelle de l'audiovisuel sans véritable statut ni salaire. Puis, nous avons obtenu un petit financement de la part de l'université qui a permis de couvrir nos frais de mission. Ce crédit a pu être obtenu à la condition d'effectuer une recherche et non pas de réaliser un film. Cette séparation, imposée par les critères de financement universitaires, ne correspondait pas à notre conception selon laquelle réalisation et recherche participent d'un même mouvement (Maillot, 2012 ; Meyer et Papinot, 2016).

D'autre part, nous avons donc dû abandonner l'idée de construire le récit du film à partir de l'expérience des migrants sur l'île. En un sens, nous nous sommes retrouvées face à un problème classique de l'enquête qualitative : lorsque la recherche s'intéresse à un phénomène d'actualité, très politisé et faisant l'objet d'interventions publiques régulières (réformes et décrets, mesures d'urgence, interventions militaires etc.), l'accès au terrain peut subitement être remis en cause (Beaud et Weber, 1997); en l'occurrence le contexte a rapidement changé (arrêt de l'accueil de migrants sur l'île) et nous nous sommes vus refuser l'accès à des lieux (par exemple, le CPSA) tandis que certaines personnes ne souhaitaient pas témoigner (invocation du droit de réserve, menace de la hiérarchie, etc.). Nous avons envisagé plusieurs options. Ce qui nous tenait, pour ainsi dire, était la volonté de restituer visuellement les spécificités de Lampedusa (l'isolement, la militarisation, le tourisme) ainsi que toute sa profondeur sociologique afin de proposer, ou suggérer, une lecture complexifiée de la situation de cette île rendue tristement célèbre. Lampedusa est à la fois une frontière politique et géographique entre l'Europe et le continent africain, et un huit clos qui contient tout ce qu'elle sépare : habitants, migrants, pêcheurs, garde-côtes, humanitaires et touristes se côtoient sur vingt kilomètres carrés sans vraiment nouer de liens réguliers et durables. Tout se passe comme si, une fois traversées les frontières officielles entre les Étatsnations, des frontières sociales et administratives invisibles séparaient les individus. 
Image 2 : des enfants jouent sur le terrain de foot, à proximité du " cimetière des bateaux "

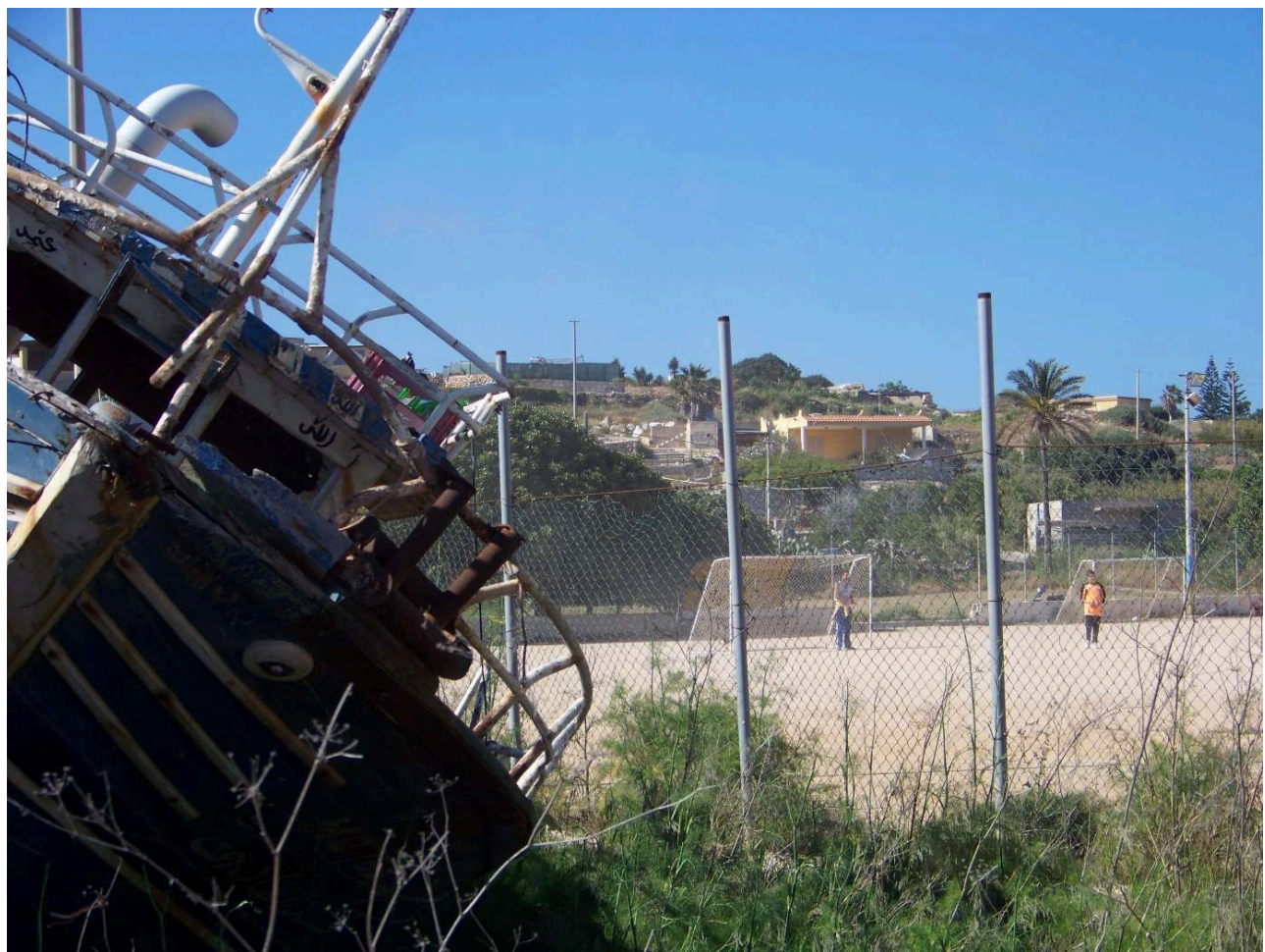

Source : Annalisa Lendaro, mai 2015

Pour faire état de ce regard singulier sur Lampedusa, nous avons envisagé deux options : d'une part, chercher à rendre ces différentes frontières visibles et, d'autre part, donner la parole aux personnes qui y vivent, y travaillent et y sont confinées. Par ailleurs, nous avions assez vite compris qu'une partie de ses habitants étaient tiraillés entre le devoir moral d'aider, en mer et sur terre, et le sentiment d'être oubliés tant par les pouvoirs publics et les médias que par les chercheurs et l'ensemble des curieux, artistes et bénévoles qui s'intéressent à leur île. Finalement, le choix de mettre au centre de la focale les voix des habitants de Lampedusa - qui semblaient être bien plus divergentes que ce que les médias pouvaient donner à penser - l'a emporté. Il y avait là, un vrai sujet sociologique dans la mesure où nous souhaitions comprendre et montrer quelles sont les dynamiques sociopolitiques et les raisons, individuelles et collectives, qui poussaient des individus à tenir et assumer publiquement des propos différents voire divergents - sur les politiques migratoires contemporaines et sur leurs effets. Autrement dit, il n'était pas seulement question de montrer que la «Lampedusa championne de solidarité » n'existe pas en tant que telle, mais il s'agissait de proposer des éléments de compréhension sociologique sur les raisons qui poussent une partie de la population à être solidaire de la cause migratoire et d'autres à être plus nuancées, voire à y être opposées ${ }^{6}$.

Nous étions tout juste au début de notre collaboration. Mon binôme était la seule à savoir filmer et prendre le son, tandis que j'étais la seule à parler l'italien et à avoir de l'expérience de la conduite d'entretiens et de l'observation sociologique. Sur le papier, la solution était simple: la distribution des rôles aurait pu suivre la ligne qui démarquait nos compétences respectives, et ainsi entraîner l'assignation de tâches d'exécution plus techniques et artistiques à ma collègue, et celles davantage 
sociologiques à moi. Ce fut d'ailleurs en partie le cas. Mais l'aspect probablement plus intéressant de ce travail à deux a été l'envie et la possibilité de se plonger dans un apprentissage mutuel. Laura m'a fait part de son savoir raconter une histoire en images, en tissant la trame du récit cinématographique par le mélange des témoignages, des atmosphères, des paysages. De mon côté, j'ai partagé mon goût pour la rencontre et pour l'écoute, en discutant avec elle de la manière de poser des questions, de relancer mes interlocuteurs afin de leur faire comprendre que leur point de vue était non seulement bienvenu mais précieux.

19 Ainsi le choix des cadrages, des lieux, des moments, du nombre et du type de prises de vues à réaliser ont été la plupart du temps effectués ensemble, en binôme. Tout comme nous avons parfois énuméré ou modifié conjointement les thèmes à aborder durant un entretien, ainsi que longuement discuté de la meilleure façon de mettre en image certaines situations observées dans les rues. Apprendre à travailler ensemble nous a demandé beaucoup de temps, déjà compté, nécessité étant d'expliquer nos avis divergents et d'échanger pour trouver un consensus. Au fond, nous restions chercheuse et documentariste. Jean Pierre Olivier de Sardan avait déjà alerté les optimistes : «Un documentariste, fut-il par ailleurs ethnologue, construit autrement son sujet qu'un chercheur, fut-il par ailleurs cinéaste » $(1994: 10)$.

20 Parfois, le besoin d'affirmer nos compétences respectives et de les voir reconnues par l'autre a pu faire émerger des tensions. Mais celles-ci ont plus fondamentalement été liées aux difficultés financières (afin de prolonger nos séjours, rémunérer correctement Laura, etc.) et donc au manque de temps qui ont caractérisé nos trois séjours de recherche ${ }^{7}$, conditions qui ont de fait impacté tant ma manière de faire de la recherche que la façon dont Laura a pu s'engager dans le travail de tournage.

21 Les temporalités de la recherche que j'avais connues ont été fortement bousculées par les impératifs du film, mais aussi en partie à cause de notre inexpérience en la matière. Nous en étions à notre premier long métrage, nous avancions entre mille incertitudes et sans un scénario déjà écrit qui aurait pu guider notre entreprise. Cela a eu une double conséquence: d'une part, nous avons collecté un grand nombre de rushs car pendant le tournage nous doutions toujours de disposer suffisamment de matériaux pour pouvoir ensuite construire notre récit filmique; d'autre part, nous avons eu tendance à rester constamment ensemble, même lors de prises de vue durant lesquelles ma présence, en tant que sociologue, n'était pas forcément nécessaire. J'étais très intéressées par l'observation du maniement de la caméra par une personne qui démontrait avoir une sensibilité esthétique remarquable. Mais cette coprésence, venant aussi du besoin d'être rassurées quant à l'accord sur le bon choix de prise, le bon moment pour le faire, la bonne personne à filmer, nous a probablement privées du temps pour collecter d'autres témoignages et filmer d'autres lieux.

Faute d'expérience et de moyens, nous nous sommes donc chargées de l'ensemble et en parallèle des aspects tant techniques et logistiques que de la recherche proprement sociologique. Mes habitudes de recherche, surtout quant à la construction de la relation enquêteur-enquêté, ont ainsi été bouleversées. Les occasions pour prendre des notes au calme ou mener des entretiens préalables avant de filmer, m'ont parfois manquées, me donnant le sentiment de "passer à côté " d'une subtilité, d'un détail peut-être important. À d'autres moments, j'ai ressenti de la gêne lorsqu'il fallait se presser pour trouver quelqu'un voulant bien nous faire part devant la caméra de ses expériences et avis sur les thèmes au centre de notre projet. Aller interpeller une personne, sans avoir 
le temps de laisser la rencontre se produire d'abord, m'a mis face à mes propres inhibitions et m'a fait prendre la mesure d'un attachement à une certaine manière de faire de la recherche.

Fort heureusement, Lampedusa est un microcosme. En raison de l'étroitesse du territoire et de la densité des interconnaissances, il est aisé de revoir les mêmes personnes à distance de quelques jours, voire de quelques heures. Ce contexte m'a souvent permis de rattraper le temps et de dépasser le sentiment de rencontres faites dans l'urgence. Par ailleurs, la grande disponibilité des personnes, acceptant de me rencontrer plusieurs fois pour livrer leur parole, d'emblée ou petit à petit, a permis de limiter les refus ou ce que j'aurais pu considérer comme des occasions perdues. Au terme des trois tournages et une fois notre projet dûment compris par nos interlocuteurs (i.e. donner la voix aux habitants de l'île, dans une démarche scientifique, donc loin de tout sensationnalisme médiatique), la plupart des enquêtés voyaient dans leur participation au film une opportunité de pouvoir s'exprimer, de se faire entendre en donnant leur avis et en partageant leurs expériences. Parfois, ils y voyaient aussi l'occasion de prendre leur revanche sur un journalisme négligeant et opportuniste.

Image 3 : des pêcheurs réparent leurs filets

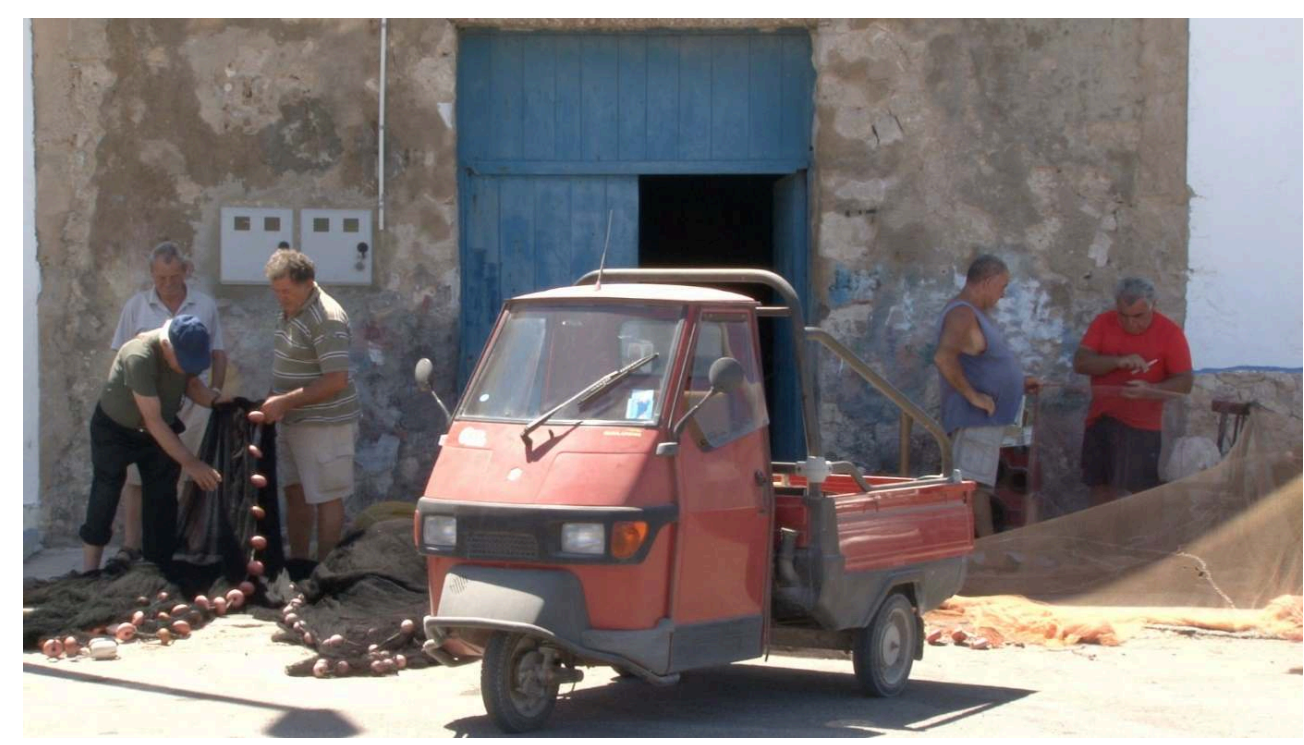

Source : Laura Auriole, août 2014

En ce sens le deuxième tournage a été déterminant, parce qu'il a eu lieu volontairement hors saison, à une époque de l'année où les habitants ne sont pas happés par les milles activités commerciales et touristiques. Le troisième et dernier tournage a également été fondamental dans la mesure où nous avons réitéré notre séjour en été, mais en l'absence des migrants : autrement dit, nous avons fait la preuve de ne pas agir tels des journalistes intéressées uniquement à couvrir un fait divers ou une énième crise institutionnelle. Nous avons ainsi gagné progressivement la confiance des habitants. Et, grâce à cette confiance, les paroles se sont libérées, y compris devant la caméra ${ }^{8}$. 


\section{Recherche et post-production : quelqu'un a dit « objectivité »?}

25 À la fin de notre troisième et dernier tournage, nous étions enfin à même d'écrire notre scénario. Avant d'avoir terminé le visionnage de l'ensemble des prises et des entretiens filmés, j'ai pris contact avec une société de production spécialisée dans l'accompagnement, la création, la diffusion et la conservation audiovisuelle de la recherche scientifique, afin de demander un soutien pour la phase de post-production.

Nous avions surtout besoin d'un monteur expérimenté capable de nous accompagner dans la phase extrêmement délicate et complexe du montage. La raison principale ne tenait pas tant à un manque de compétences techniques qu'au besoin de bénéficier d'un regard neuf, vierge de toute expérience de tournage, et mieux en mesure de contribuer à la construction du récit de manière à le rendre intelligible à tous, y compris - voire surtout - aux personnes ne connaissant pas la thématique ni le contexte spatial et sociopolitique de Lampedusa. Pendant cette phase d'écriture en duo, préalable au travail avec la société, il a notamment été question de faire des essais de montage sur des courtes scènes qui nous semblaient importantes et d'organiser des petites séances de projection. Alors que pour nous, le message véhiculé par ces séquences était limpide, il l'était beaucoup moins pour ces premiers spectateurs (amis et collègues diversement familiarisés avec le langage cinématographique). Ceux-ci nous ont fait comprendre que ces séquences n'étaient pas intelligibles pour des personnes ne bénéficiant pas de diverses informations issues de l'expérience de terrain.

Nous avons alors soumis une demande de financement avec un dossier présentant le projet et mettant l'accent sur notre démarche combinant sociologie et cinéma. Durant l'été 2015, nous avons rencontré une première fois le responsable de la production ainsi qu'un des réalisateurs de la structure, et sommes d'emblée rentrées dans des négociations autour du futur film. L'intérêt pour cette société de produire Benvenuti était forte, mais la durée que nous avions initialement prévue (90 minutes) dépassait ce que la production estimait devoir être la durée de notre long-métrage : « Moins de 60 minutes, à prendre ou à laisser ». Nous avons accepté, convaincues par l'argument selon lequel une telle durée permettrait une diffusion du film plus ample. Nous nous engagions ainsi à collaborer avec une équipe à laquelle nous avions clairement exprimé notre objectif : réaliser un film de recherche capable d'interpeller le spectateur, afin de diffuser la connaissance mais aussi de provoquer la curiosité, le débat, voire l'émoi. Il y avait là, surtout pour moi, l'envie de bénéficier d'une nouvelle liberté d'expression, bien moins contrainte que celle de l'écrit académique. Aussi je m'attendais à être non seulement explicitement autorisée - voire encouragée - à créer des atmosphères, à m'appuyer sur des émotions, à faire naître le doute, mais également à fournir des clés d'interprétations avec l'objectif de donner au spectateur l'envie d'aller au-delà des apparences, sans trop le guider ou lui imposer une pensée.

Mais les négociations avec la société de production ont été plus rudes que ce que j'imaginais. Tout d'abord, il a été question de discuter de l'intérêt d'avoir un commentaire en voix off. En l'absence d'un personnage principal et dans le but de réussir le pari du transfert de connaissances vers un public de non spécialistes, la production a longtemps insisté pour qu'une voix off intervienne régulièrement, faisant office de médiation pédagogique afin de guider le spectateur dans la compréhension du projet et de ses résultats. Toutes deux prônions en revanche l'absence de voix off, 
procédé que nous considérions comme le véhicule d'interprétations "prêtes à l'emploi» (Giglio-Jacquemot 2013). Nous voulions faire confiance aux images et au travail de montage en cours, mais surtout nous considérions que le contenu des séquences filmées permettrait de fournir des outils d'interprétation suffisants à la circulation des connaissances (comme la combinaison réfléchie d'extraits d'entretiens par exemple). Autrement dit, nous défendions une idée différente du spectateur : nous le pensions comme un être autonome à qui nous voulions donner l'envie de se questionner, de se renseigner et de se méfier des partis pris, plutôt que des explications. Nous sommes parvenus à convaincre la société et aucune voix off n'a été ajoutée.

En même temps, il a fallu négocier autour d'un autre aspect fondamental de ce travail collectif.

À plusieurs reprises, une question est revenue pendant les discussions : « Quels sont et où se trouvent les résultats de la recherche dans le film?». On nous demandait que le film témoigne de la scientificité de la démarche. Petit à petit nous avons compris : pour nos interlocuteurs le sérieux d'une recherche et son degré de scientificité étaient considérés comme incompatibles avec les traces de notre sensibilité politique et de notre prise de position sur un sujet d'actualité si controversé. La société ne semblait pas favorable aux critiques et débats contradictoires que pouvait induire de telles prises de positions. Si pour notre binôme de sociologue et de réalisatrice, la confrontation des points de vue correspondait à notre idée de la recherche et à nos ambitions initiales, pour la production, le film aurait dû servir à présenter et à rendre intelligibles les résultats de la science, à instruire et convaincre le public de manière incontestables.

Image 4 : restes de bateaux de migrants sur une zone miliaire

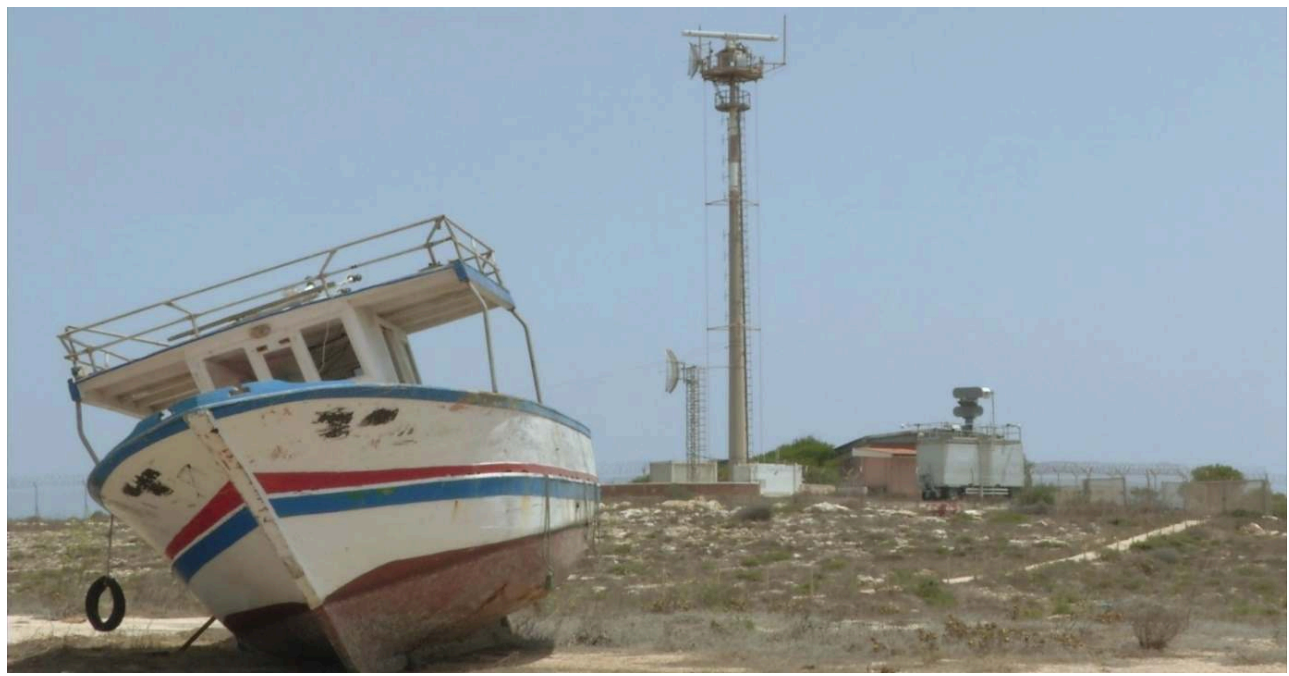

Source : Guillaume Bordier, juillet 2014

31 Cette opposition s'est matérialisée lors des projections des versions de montage intermédiaires. Nous travaillions constamment à trois, les deux réalisatrices et le monteur professionnel, puis nous organisions des réunions avec l'équipe de la production pour engager la discussion et valider ou amender les propositions. À ces occasions, des requêtes explicites nous ont été formulées : ne pas utiliser certains rushs, réduire la durée de certaines scènes ou en changer le rythme, flouter des images. Un 
argument puissant a été utilisé : il aurait fallu obtenir les autorisations officielles de l'armée de terre italienne et de la Préfecture pour pouvoir utiliser des clips montrant les casernes, les radars, les véhicules des forces de l'ordre, même si les images avaient été filmées dans l'espace public. La société refusait, non pas de s'engager dans une improbable procédure juridique liée au droit à l'image, mais plutôt d'endosser la responsabilité morale d'avoir produit un film dont des personnes et des institutions auraient pu reprocher un engagement politique «incompatible avec la science». Les deux parties ont fini par faire des concessions et nous nous sommes accordés sur une version finale $\mathrm{du}$ film. Benvenuti est sorti fin 2016 avec une première au festival Cinemed de Montpellier. Cependant, suite à l'obtention d'un prix à la diffusion ${ }^{9}$ reçu en novembre 2017 lors du Festival de la Méditerranée en images, la société s'est opposé à la diffusion télévisuelle du film et à son inscription dans des festivals de cinéma. Les deux réalisatrices ont fini par demander et obtenir la rétrocession intégrale des droits sur le film en 2018. Cette expérience, que je ne regrette nullement pour autant, m'a donné envie de repartir sur le terrain et de faire vivre le film autrement.

\section{Retour sur le terrain : le film comme outil de recherche}

Le bousculement du temps de l'enquête ethnographique, au profit d'un rythme trop souvent dicté par les opportunités de filmer des instants ou des personnes, figure parmi les malaises déjà évoqués précédemment. Une fois le film terminé, j’ai commencé à songer à retourner sur le terrain, sans caméra et en marge de tout projet de diffusion $\mathrm{du}$ film. Je souhaitais replonger dans les temps longs des interactions interpersonnelles, et donner à voir le résultat de ce travail mené au long de trois années aux personnes les plus directement concernées. J'ai alors utilisé le film comme outil pour l'enquête. Entre avril et juin 2018, quatre ans après le dernier tournage, j'ai organisé la projection du film dans le cadre d'entretiens collectifs ${ }^{10}$ avec un certain nombre d'habitants de l'île de Lampedusa. Cette expérience étant encore récente et nécessitant de plus amples réflexions, je propose modestement ici de présenter quelques réflexions à chaud concernant la restitution de l'enquête comme participant d'une éthique professionnelle et les enjeux liés à un usage empirique du film comme outil de recherche.

À l'origine j'envisageais de considérer le visionnage collectif du film comme une expérience partagée - entre les participants et l'enquêteur, et entre les participants eux-mêmes - favorisant l'expression et la confrontation de points de vue (mais aussi dans le cadre d'entretiens individuels successifs; je reviendrai sur ce point) (Papinot 2016). En cela, le film peut participer d'une modalité assez peu utilisée en vue de l'obtention de données qualitatives. Avant même de se questionner sur le type et la pertinence des données récoltées par cette pratique, il y a à mes yeux un avantage indéniable : répondre à l'exigence de restituer le résultat du travail scientifique en proposant un droit de regard aux personnes concernées. Si certaines contributions et manuels de méthode rappellent l'intérêt de la restitution (Kobelinsky 2008, Bergier 2000, Beaud et Weber 1999, entre autres), elle n'est néanmoins pas systématiquement adoptée en sociologie. Souvent, la forme que prend la valorisation des résultats d'une recherche (articles et ouvrages spécialisés, mémoires, thèses, essais ou rapports plus ou moins techniques) est difficile d'accès pour un public non spécialisé ou familier du langage scientifique, voire totalement inaccessible pour les non locuteurs de langue dans laquelle publie le chercheur. Le film documentaire est en ce sens une forme de 
rendu de la recherche considérée par les enquêtés eux-mêmes, à raison selon moi, comme plus accessible et donc mieux à même de permettre de réagir et de formuler des avis.

Prendre au sérieux le devoir de restitution des résultats de l'enquête aux participants revient à traduire en actes une certaine éthique du travail de terrain, basée sur l'idée d'un échange entre chercheurs et participants. Ces derniers, loin d'être considérés uniquement comme des sources d'information et de matériaux (oraux, iconographiques, etc.), sont alors impliqués dans la discussion des interprétations, raisonnements et analyses ayant contribué à la compréhension du phénomène ou du processus étudié. La compréhension du chercheur est donc mise à l'épreuve collective de la restitution et du droit de regard des participants aux projections.

Le film peut dès lors avoir une utilité méthodologique non négligeable. Le fait de pouvoir discuter collectivement des résultats d'une première phase d'enquête par le biais du visionnage du documentaire permet l'émergence de nouvelles pistes de recherche et de nouvelles interprétations, favorisant ainsi une compréhension potentiellement plus fine des processus considérés. Présentée en début d'entretien collectif, cette démarche a aussi l'intérêt d'atténuer la distance entre l'enquêteur et les participants : ces derniers sont explicitement invités à commenter et critiquer le travail du premier, et ainsi contribuer à le faire progresser dans sa recherche en bénéficiant de divers points de vue.

Inspirée d'une approche pragmatique de l'enquête (Dewey 2010, Quéré 2012, Ogien 2014) - attentive aux expériences et aux significations mouvantes et contextuelles, tant de l'enquêteur que des enquêtés -, ces discussions ont été l'occasion d'observer comment et à quelles conditions les considérations sur des questions sensibles comme l'immigration s'expriment, se modifient, se nuancent ou sont passées sous silence. Les échanges collectifs faisant suite aux projections ont parfois été associés à des entretiens individuels avec certains participants ${ }^{11}$. J'ai alors souhaité orienter le moins possible ces entretiens avec l'objectif de dépasser, autant que possible, la dichotomie vrai-faux (Karsenti et Quéré 2004) afin de saisir «ce à quoi nous tenons» (Bidet et al. 2011), individuellement et collectivement, et pourquoi.

37 J'ai convié diverses personnes rencontrées préalablement sur le terrain, et appartenant à différents groupes sociaux internes à l'île, à participer aux projections tout en leur proposant d'inviter d'autres connaissances. Ces personnes avaient soit participé au film, soit discuté du projet avec nous depuis les premiers tournages. La tendance, prévisible, à inviter les personnes considérées comme étant les plus qualifiées ou intéressées parmi leurs connaissances a été tempérée par mes propres invitations adressées à des personnes externes au groupe d'appartenance des personnes ressources. Dès lors, les participants n'avaient pas l'appartenance à une catégorie socioprofessionnelle ou à une tranche d'âge en commun, mais l'expérience de l'insularité et d'une gestion hors norme du phénomène migratoire sur leur territoire. 
Encadré 1 : les entretiens collectifs réalisés

\begin{tabular}{|c|c|c|c|c|c|}
\hline Lieu & $\begin{array}{l}\text { Salle } \\
\text { commune du } \\
\text { Centre pour } \\
\text { malades } \\
\text { psychiques }\end{array}$ & Bibliothèque & $\begin{array}{l}\text { Salle d'une } \\
\text { Fondation } \\
\text { participant } \\
\text { aux secours } \\
\text { en mer }\end{array}$ & $\begin{array}{l}\text { Magasin du } \\
\text { village }\end{array}$ & $\begin{array}{l}\text { Appartement } \\
\text { privé }\end{array}$ \\
\hline $\begin{array}{l}\text { Nombre de } \\
\text { participants }\end{array}$ & 17 & 12 & 5 & 5 & 6 \\
\hline $\begin{array}{l}\text { Composition } \mathrm{du} \\
\text { groupe }\end{array}$ & $\begin{array}{l}\text { Hétérogène: } \\
\text { opérateurs et } \\
\text { usagers du } \\
\text { centre, } \\
\text { bénévoles } \\
\text { d'une } \\
\text { structure } \\
\text { associative, } \\
\text { professions } \\
\text { libérales } \\
\text { sensibles à la } \\
\text { question de } \\
\text { l'accueil des } \\
\text { migrants }\end{array}$ & $\begin{array}{l}\text { Plutôt } \\
\text { hétérogène : } \\
\text { bénévoles de } \\
\text { la } \\
\text { bibliothèque, } \\
\text { président de } \\
\text { l'archive } \\
\text { historique, } \\
\text { prêtre, et } \\
\text { gérant du } \\
\text { camping }\end{array}$ & $\begin{array}{l}\text { Homogène: } \\
\text { toutes et } \\
\text { tous } \\
\text { bénévoles de } \\
\text { la fondation, } \\
\text { en tant que } \\
\text { médecins ou } \\
\text { infirmiers. }\end{array}$ & $\begin{array}{l}\text { Plutôt } \\
\text { hétérogène : } \\
\text { Deux } \\
\text { commerçants } \\
\text { et trois amis- } \\
\text { clients du } \\
\text { magasin }\end{array}$ & $\begin{array}{l}\text { Homogène: } \\
\text { trois } \\
\text { opérateurs du } \\
\text { CSPA, et des } \\
\text { membres de } \\
\text { leurs familles }\end{array}$ \\
\hline $\begin{array}{l}\text { Degré } \\
\text { d'interconnaissance }\end{array}$ & Plutôt faible & Fort & Moyen & Très fort & Très fort \\
\hline $\begin{array}{l}\text { Entretiens } \\
\text { individuels } \\
\text { complémentaires }\end{array}$ & 5 sur 17 & 4 sur 12 & Non & 1 sur 5 & 3 sur 6 \\
\hline
\end{tabular}

Qu'est-ce qui se partage lors des discussions issues de ces entretiens? Qu'est-ce qui divise ? Qu'est-ce qui n'est pas exprimé lors d'une discussion collective mais peut l'être dans le face à face avec l'altérité du chercheur? L'analyse de l'ensemble des données étant encore en cours, je botterai en touche à ce stade sur les réponses à ces questions. En revanche, elles me permettent de revenir sur quelques procédés adoptés afin de me donner les chances de pouvoir y répondre.

J'ai accordé une importance particulière à la présentation du dispositif des entretiens, dans le but d'expliquer, à celles et ceux qui ne connaissaient pas en amont le projet de film, son origine, ses développements successifs ainsi que les raisons me poussant à montrer le résultat de notre enquête. La discussion n'a pas été enregistrée, j'ai pris des notes pendant et après nos réunions.

40 À la suite de chaque projection, la discussion a systématiquement démarré autour du film : j'ai alors été questionnée par les enquêtés, j'ai pris activement part à la discussion pour apporter des réponses et soulever des questions à mon tour. J'ai parfois sollicité les participants à changer de point de vue: "Qu'auriez-vous fait à ma place?» «Quelles images et quels entretiens auriez-vous choisi pour dire cela?» 

ou pas abordées dans le film, et importantes à leurs yeux : le rapport de tout un chacun au respect de la norme, la méfiance envers les institutions publiques, les changements économiques majeurs ayant impacté l'île. Ainsi orienté, la discussion ne s'appuyait pas sur une grille d'entretien préparée au préalable, puisqu'il procédait d'une adaptation in itinere aux interventions des participants. Les expériences de ces derniers étant au cœur des échanges, j'ai moins pris la parole, prenant le maximum de notes sur leur contenu et les interactions non verbales (regards, soupirs, grimaces, etc.).

Image 5 : Projection lors d'un entretien collectif

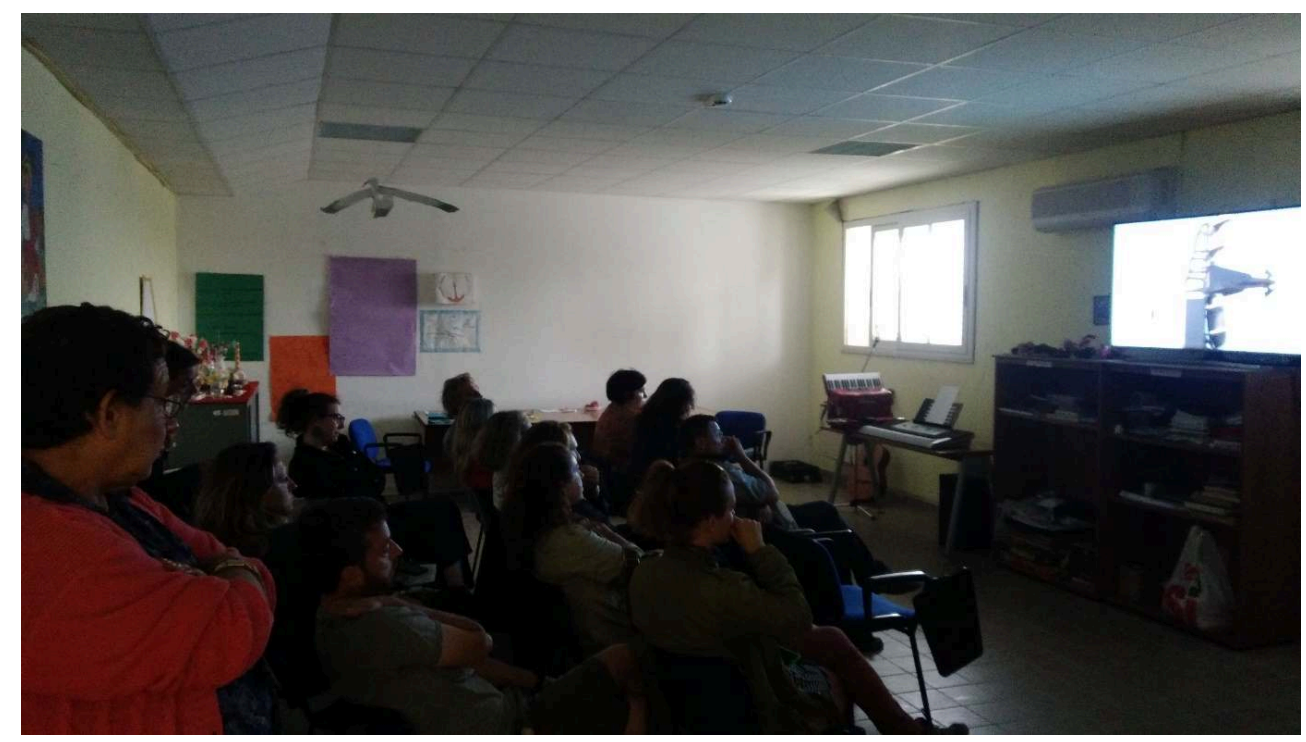

Source : Annalisa Lendaro, mai 2018

La participation aux discussions collectives et l'observation des formes de communication discrètes (silences, hésitations etc.) m'ont permis de comprendre que la parole n'est pas toujours libre de s'exprimer selon les interlocuteurs présents et les sujets abordés. Certains participants savent ou supposent ne pas partager le même avis que d'autres sur des thèmes délicats et politisés. Qu'est-ce qui fait que ce dissensus éventuel se manifeste en situation d'entretien collectif ? Qu'est-ce qui l'atténue ? Le fait de noter qui prend la parole et qui reste en silence a été certainement plus utile que de tenter d'équilibrer le temps de parole entre les participants.

Les entretiens individuels faisant suite aux discussions collectives ont permis de mieux comprendre les absences et les silences ou, au contraire, les prises de parole fréquentes et l'assurance de certains lorsqu'il s'agissait d'aborder tel ou tel sujet. En établissant le profil sociologique et la place sociale (acquise, souhaitée, récusée...) des individus présents, j'ai alors mieux compris la dynamique relationnelle au sein de cette petite communauté caractérisée par une interconnaissance forte et des enjeux de réputation marqués. Si parler de politique ou évoquer l'accueil des migrants en public est perçu comme un danger - parce que la personne anticipe le conflit avec d'autres participants à la discussion -, il devient en revanche possible d'obtenir des informations lors d'échanges discrets menés avec une chercheuse étrangère à la communauté : «C'est bien parce que tu n'es pas d'ici que je peux te dire ce que je pense !». 
deuxième vie du film documentaire marque paradoxalement mon retour à l'écrit. Sans regrets particuliers au vue du goût retrouvé pour le détail que l'écriture permet de restituer. Reste cependant pour moi toujours ouverte la question de la diffusion et du partage de la recherche : parler aux pairs, être compris et même critiqué par eux, est légitime et valorisé dans le monde universitaire. Il me semble néanmoins nécessaire de trouver un compromis pour à la fois rendre justice à la complexité des terrains et des raisonnements analytiques, et les rendre accessibles à un public plus large.

\section{BIBLIOGRAPHIE}

Beaud S. et Weber F. (1997), Guide de l'enquête de terrain. Produire et analyser des données ethnographiques, Paris, La Découverte.

Bergier B. (2000), Repères pour une restitution des résultats de la recherche en sciences sociales. Intérêts et limites, Paris, L'Harmattan.

Bidet A., Quéré L., et Truc G. (2011), John Dewey, La formation des valeurs, Paris, La Découverte, p. 5-64.

Cuttitta P. (2015). « La 'frontiérisation' de Lampedusa, comment se construit une frontière », L'Espace Politique, vol. 25, n 1, mis en ligne le 08 avril 2015, consulté le 05 juillet 2016.

Cuttitta P. (2012), Lo spettacolo del confine, Milano, Mimesis.

Dewey J. (2010), Le public et ses problèmes, Paris, Gallimard.

Duschesne S., Haegel F. (2004), L'enquête et ses méthodes : l'entretien collectif, Paris, Armand Colin.

Giglio-Jacquemot A. (2013). «Descriptivité et émicité du documentaire : les choix de réalisation d'un film sur le travail domestique ». Ethnographiques.org, $\mathrm{n}^{\circ}$ 25, URL : http:// www.ethnographiques.org/2012/Giglio-Jacquemot\#silence-commentaire-8

Karsenti B et Quéré L. (dir.), 2004, La Croyance et l'Enquête, Paris, Éd. de l'EHESS.

Kobelinsky C. (2008), « Les situations de retour. Restituer sa recherche à ses enquêtés », Les politiques de l'enquête. Epreuves ethnographiques. Paris, La Découverte, p. 185-204.

Lendaro A. (2018).

Maillot P. (2012). « L'écriture cinématographique de la sociologie filmique. Comment penser en sociologue avec une caméra? ", La Nouvelle Revue du travail [En ligne], vol. 1, mis en ligne le 10 décembre 2012, consulté le 20 juillet 2014. URL : http://nrt.revues.org/363

Melot É. (2009), « Lampedusa, terre de confins », Mouvements, nº 60, p. 124-129.

Meyer M., Papinot C. (2016). « Le travail des images dans la démarche de recherche. Analyse réflexive et compréhension de l'objet ", Images du travail, travail des images, $\mathrm{n}^{\circ}$ 3, URL : http:// imagesdutravail.edel.univ-poitiers.fr/index.php ?id =1120

Nicolosi G., Panebianco K. (2015). « Les morts de Lampedusa », Communications, vol. 97, n² 2, p. 161-176.

Images du travail, travail des images, 8 | 2020 
Ogien A. (2014), « Pragmatismes et sociologies », Revue française de sociologie, vol. 55, $n^{\circ} 3$, p. 563-579.

Olivier de Sardan J. P. (1994), Pacte ethnographique et film documentaire, Xoana-Images et sciences sociales, $\mathrm{n}^{\circ}$ 2. URL : http://imagesdutravail.edel.univ-poitiers.fr/index.php ?id =1230

Papinot C. (2016). « La machine à café, l'atelier et la chaîne. Quelques réflexions sur l'usage de la photographie comme support d'entretien ", Images du travail, travail des images, n 3 , URL : http:// imagesdutravail.edel.univ-poitiers.fr/index.php ?id =1215, consulté le 5/5/2018

Quéré L. (2012), « Pragmatisme et enquête sociale », La théorie sociale contemporaine, Paris, Presses universitaires de France, p. 182-194.

Ritaine É. (2015), « Quand les morts de Lampedusa entrent en politique : damnatio memoriæ », Cultures \& Conflits, $n^{\circ}$ 99-100, p. 117-142 .

Rodier C. (2014), « Naufrage de Lampedusa », Multitudes, n 55, p. 20-24.

\section{NOTES}

1. Ce point fait l'objet d'un développement spécifique dans l'article.

2. Aujourd'hui, plus que jamais, une partie de la presse et des productions télévisuelles est responsable à mes yeux d'entretenir des discours et des représentations faussées et instrumentalisées de phénomènes ou événements en lien avec la migration. Les exemples de messages simplificateurs sont nombreux : associer le terme de passeur à des ONG opérant en Méditerranée, parler de "révolte des clandestins dans les centres d'accueil » sans préciser les conditions de cette détention (comme la mixité d'adultes et d'enfants non accompagnés) et s'ils ont le droit de demander l'asile, ni préciser que les autorités n'accordent pas à ces personnes la possibilité d'enregistrer cette demande et qu'elles sont souvent maintenues en détention administrative pour des périodes dépassant largement la durée légale prévue par la loi, etc. Ces affirmations, fréquemment diffusées en Europe, ont une portée politique incontestable et contribuent à nourrir un climat de peur, de méfiance, voire de xénophobie.

3. Il s'agit ici d'un écart à la règle plutôt ordinaire à Lampedusa. En fermant les yeux, les forces de l'ordre rendaient la frontière du centre poreuse, notamment car lorsque le nombre de personnes à y être enfermées est trop élevé, c'est un moyen de réguler les conflits et les risques d'épidémie.

4. Pour une présentation succincte de l'opération voir: http://www.marina.difesa.it/EN/ operations/Pagine/MareNostrum.aspx

5. Une vidéo tournée par un migrant au sein du CPSA fait la une des médias européens entre décembre 2013 et janvier 2014 https://www.nouvelobs.com/monde/20131218.0BS9892/ lampedusa-la-video-de-la-honte.html

6. Pour reprendre les termes d'olivier de Sardan, nous souhaitions « fournir un accès minimal et authentifié à la "vision du monde" des sujets filmés ", ce qui revenait à assumer la "spécificité du pacte ethnographique, dont on voit bien qu'elle correspond à l'une des dimensions majeures du propos anthropologique en général (en particulier ce souci de la restitution "émique" des comportements et des faits sociaux)» (1994:8).

7. Dix jours de repérages ont été autofinancés en 2013, puis quinze jours et un mois de recherches-tournages ont été financés par des missions en 2014.

8. Dans le souci de ne pas idéaliser et attribuer une sorte de systématicité à la parole libérée, il me semble pertinent de préciser que, à plusieurs reprises, nous avons remarqué un changement de ton ou même de contenu dans la parole enregistrée devant la caméra par rapport à celle livrée hors enregistrement. Il a été aussi instructif par la suite de provoquer la discussion avec ces 
personnes autour de ce qui se dit et ce qui ne se dit pas en public, dans le cadre d'un dispositif filmique. Il y a là, il me semble, un aspect heuristique non négligeable dans la combinaison de l'outil filmique et de l'entretien classique.

9. Le prix consistait en une diffusion télévisuelle sur la chaine France 3 Via Stella.

10. Selon la définition qu'en donnent Florence Heagel et Sophie Duchesne (2004), l'entretien collectif fait référence a minima à un échange qui implique au moins deux personnes et met en jeu une relation sociale dépassant le traditionnel couple constitué par l'enquêteur et l'enquêté. En termes de composition, si ces entretiens peuvent concerner des groupes au sens sociologique $\mathrm{du}$ terme, ils peuvent aussi rassembler des individus qui ne se connaissent pas ou ne partagent pas - ou peu - de caractéristiques communes.

11. Des entretiens individuels ont été menés avec treize des quarante-cinq participants ayant participé aux cinq entretiens collectifs. Trente-deux entretiens individuels supplémentaires ont été réalisés avec des personnes n'ayant pas participé aux entretiens collectifs.

\section{RÉSUMÉS}

Cette contribution raconte l'histoire d'un film documentaire, à partir des conditions de sa réalisation jusqu'à ses usages ex-post. Benvenuti $(53$ ', 2017), coréalisé par une sociologue et une documentariste, est le fruit d'une recherche sur les frontières matérielles et symboliques qui traversent une petite île italienne: Lampedusa. Dans cet article, en m'appuyant sur un retour réflexif d'expérience, je tente de répondre à la question suivante : à quel moment du processus de recherche l'image animée joue-t-elle un rôle central dans la compréhension d'un phénomène social et dans la production scientifique ? Après avoir retracé les moments clés de ce parcours à deux et leurs enjeux (l'origine du projet, les tournages, la post-production), je me concentrerai sur l'intérêt que représente le film en tant qu'outil d'enquête : en particulier, il sera question de discuter de l'usage de sa projection dans le cadre d'entretiens collectifs réalisés entre avril et juin 2018 lors d'un retour sur le terrain, de la seule sociologue cette fois-ci.

This article tells the story of a documentary film, from its shooting to its screenings. Benvenuti $(53,2017)$ was co-directed by a sociologist and a documentary maker, and is the fruit of a research on the borders and boundaries that penetrate a small italian island : Lampedusa. In this contribution, through a reflexive return to the fieldwork, I try to aswer the following question : at what stage(s) of the research work does the animated picture play a central role for the understanding of a social phenomenon? After having reconstructed the different phases of its realisation, I will focus on the methodological issues dealing with a reasearch film : in particular, I will discuss the pertincence of its screening as a part of a set of collective interviews relizes between april and june 2018 during a return on the same fieldwork (the sociologist alone, this time.

\section{INDEX}

Mots-clés : film documentaire, Lampedusa, entretiens collectifs, restitution d'enquête Keywords : documentary film, Lampedusa, collective interviews, survey report 


\section{AUTEUR}

\section{ANNALISA LENDARO}

Chargée de recherches en sociologie politique au CNRS (France). Ses principaux intérêts portent sur les politiques migratoires, leurs applications dans les territoires frontaliers et leurs effets sur l'accès aux droits et sur les mobilisations collectives. En utilisant des méthodes ethnographiques, ses études essaient de mettre en lumière les processus qui rendent ordinaire le contournement du droit. Annalisa est la coordinatrice de l'ANR DisPow (2018-2021), un projet qui se propose d'étudier les formes et les effets du pouvoir discrétionnaire de l'État à trois frontières française : le Calaisis ; la zone se situant entre Vintimille, Bardonecchia, Briançon et Nice ; et la frontière basque entre Bayonne et Irun. 